\title{
Perceived rotary motion from changes in a straight line
}

\author{
GUNNAR JOHANSSON 1,2 AND GUNNAR JANSSON \\ DEPARTMENT OF PSYCHOLOGY, UPPSALA UNIVERSITY, Sweden
}

The problem dealth with was how perception of a threedimensional space is related to the corresponding two-dimensional retinal image. The stimulation used was a straight line changing length and direction. For this stimulation a geometrical model was developed. The basic decoding principle in this model is that the changes in the two-dimensional figure will be perceived as three-dimensional motions of an object with constant shape and size. This principle was approximately verified for the majority of the data. The main deviation from the model was that there was generally less perceived depth than predicted. Also a second decoding principle was generally verified: rotary, but not translatory motion is perceived from this kind of stimulation. A third unexpected decoding principle was found in the data: the line is perceived in a frontal-parallel direction when it has its maximal extention.

How are specific percepts of objects in a three-dimensional space obtained? The problem about three-dimensional percepts from two-dimensional retinal images has been a famous one since Berkeley (1709) and it is, in fact, not yet satisfactorily solved. Berkeley's discussion and most of the later treatments of the problem dealt with percepts from static stimulation. Such stimulation is a rare condition, however, and percepts from static stimulation may be regarded as a special case with percepts from changing stimulation being the general case. The investigation to be reported here constitutes a part of a research program about space and object perception from this more general kind of stimulation.

In an attempt to start a systematic analysis of the stimuluspercept relationships in the visual perception of motion and/or change of shape the senior writer recently studied some patterns of changing stimulation (Johansson, 1964). As independent variables in these experiments were chosen changes in the proximal stimulation, described as changes in the distribution of light on a picture plane. These patterns were generated electronically, and in most cases they were not possible to describe as projections of rigid objects in motion. This approach thus differed from that of the earlier studies of changing stimulation using a shadow caster technique with rigid real objects in motion as shadow generators (e.g., Gibson \& Gibson, 1957). It also differed from that of Green (1961) and Braunstein (1962), as their proximal stimulation consisted of polar projections of imagined distal objects.

In the senior writer's earlier study the stimuli used were rectangular areas changing size and shape. For geometrical reasons there is no specific information about shape changes and/or motions of an object inherent in the proximal stimulation. The percept is specific, however, and this seems to indicate that the visual system applies some decoding principles in order to get specific percepts. Therefore, a basic series of experiments was carried out openended, i.e., no hypothesis was raised as to the perceptual outcome from the stimulation. The main task was a systematic search for a general mathematical analogue to the perceptual outcome from the stimulus patterns. It was possible to abstract a few basic decoding principles which when applied to actual stimulus patterns resulted in descriptions analogous to the percepts.

The mathematical analogue consisted of a geometrical model with (1) a station point corresponding to the eye, (2) a projection plane, corresponding to the picture plane, and (3) a projective space, into which a change corresponding to the proximal stimulation was projected from the projection plane by reverse polar projection. The model was concerned only with monocular vision.

The decoding principles found may be simplified and summarized in this way: Three-dimensional motion is usually per- ceived, and the object executing this motion is perceived as rigid as the stimulus pattern allows.

In a more informal continuation of these experiments changing angles in parallelograms were studied. The resulting percepts were always described as rotations or tilting movements of a rigid surface. These effects constituted the starting point for the experiments which are reported in the present paper. An extremely simple case of spatial change was investigated in these experiments: changes of length and direction of a straight line against a totally dark background. This kind of stimulation has also been studied by Wallach and O'Connell (1953). These authors studied different kinds of stimuli and drew the conclusion that lines changing length and direction constituted an essential condition for the occurrence of the kinetic depth effect. The generality of this principle was questioned by White and Mueser (1960).

A model similar to the model outlined for rectangular areas was developed for this simple proximal stimulation. The general problem for the experiment described here was to study the applicability of this model.

\section{THE MODEL}

\section{The Projection System}

A model, similar to the model earlier outlined by the senior writer (Johansson, 1964) was elaborated in the following way in order to make it possible to obtain quantitative predictions of percepts from the stimulus patterns studied here.

Figure 1 shows a diagram of an eye (S) with the direction of gaze indicated by $Z$. This line is the $Z$-axis in our projection system, and as an alternative name we will use the term sagittal axis. The sagittal axis cuts the X-Y-plane at right angles and in the origin of the three-dimensional coordinate system. The X-Y-plane forms the picture plane in our system, and the space defined by this system will be termed the projection system. On the picture plane there is a line drawn through origin. This line represents the stimulus line in our experiments. It will continuously change its length symmetrically from both ends and its direction, i.e., rotate around the origin over an angle $<90$ deg.

Figure 2 shows a part of the picture plane with the two turning positions of the upper half of the stimulus line $\left(a_{1}\right.$ and $\left.a_{2}\right)$. The line is moving over the angle $\alpha_{2}-\alpha_{1}$ and is at the same time changing length as indicated by the dotted line. In our following analysis these lines will be specified by their polar coordinates in the plane $(a=$ length of half the stimulus line and $\alpha=$ angle in relation to the $\mathrm{X}$-axis). The lower part of the stimulus line moves

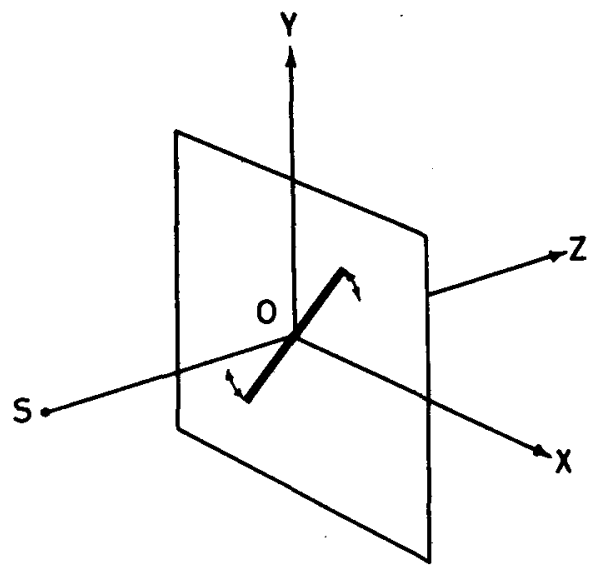

Fig. 1. The projection system. 


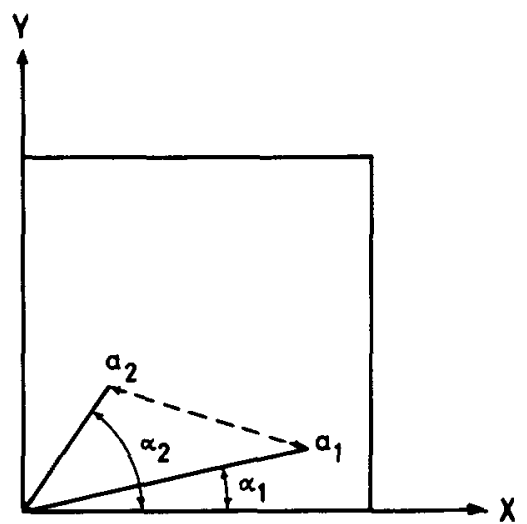

Fig. 2. Part of the picture plane with the turning positions of the upper part of the stimulus.

in a completely symmetrical manner, the whole stimulus line being straight all the time.

\section{Possible Projections}

This changing line will now be thought of as projected into the projection space. In order to make the description simple we will, in the first part of our analysis, exclude the rotary component and only treat the effect of changing length of the line. We find that there will be an infinite number of geometrically correct projections. Because we have allowed only change of length of the line, all these projections will lay on a stationary sagittal plane through the line. The projection is always thought to be a straight line. Let us categorize the possible projections in the following five categories:

(1) The length of the line is changing in a plane parallel to the picture plane.

(2) The line has a constant length and executes a rotary motion in the projection space with a determined point on the sagittal axis as its center of rotation. Its angle to the sagittal axis is not determined and, consequently, neither is its length. Two possible combinations of length and angle of motion of the projected line are shown in Fig. 3.

(3) The line has a constant length and describes a translatory motion backwards in the projection space, all the positions being parallel to each other. See Fig. 4. Also in this case information about the angle to the picture plane and about the length is lacking. Therefore, the line $\mathrm{a}$ is equally well represented by $\mathrm{P}$ as by $\mathbf{P}^{\prime}$.

(4) The line, its length being constant, executes any of an infinite number of combinations of translatory and rotary motions, the center of rotation moving along the sagittal axis.

(5) The line executes any of an infinite number of combinations of translatory and rotary motions as under (4) above, but changes also its length.

Now we will complete the analysis by adding the component of rotation onto the line on the picture plane. From the point of view of projection this is a far simpler affair. The components of rotation of the line about origin may be described as a rotation about the sagittal axis of the sagittal plane carrying any of the projected motions or changes described above. In Fig. 5 two such planes are shown, separated by an angle of $180 \mathrm{deg}$. For the component of rotation there is always specific information in the stimulus, and therefore we will hypothesize that in the perceived motion it will be represented in a mathematically correct way.

\section{Decoding Principles}

In accordance with the main outcome in the senior writer's earlier study, discussed above, we will set forth the following basic principle for decoding the nonspecific component of change of length: Changes in the two-dimensional figure on the picture plane will be perceived as motions of an object with constant shape and size. As a consequence of this decoding principle only those projections are accepted as possible percepts where the line is straight and has a constant length, and thus all changes, are transferred to motion. Thus, category (1) and (5) above are outside the domain of our model.

Our second principle states that rotary, but not translatory motions are perceived. For geometrical reasons a change in the angular coordinate on our picture plane cannot be projected as a translatory motion, but only as a rotation. Neither had the pre-experiments brought about any percepts involving translatory motion. Therefore, for the sake of simplicity, translatory motion was excluded from the present model. The effect of this decision was that the categories (3) and (4) were made invalid, and our preliminary model will deal only with category (2), i.e., rotations in a three-dimensional space of a line of constant length.

\section{Trigonometric Analysis}

Figure 5 shows a sagittal plane in the projection system. The plane extends on both sides of the $\mathrm{Z}$-axis and it passes through the stimulus line on the picture plane at the two turning positions during the cycle of change. Thus, the angle between the lines on the picture plane $\left(\alpha_{2}-\propto_{1}\right)$ is in this special case $=180 \mathrm{deg}$. This angle size was chosen here for technical diagrammatical reasons, and the relations to be stated are valid for any value of this angle. For the sake of simplicity only half the stimulus line is given in the figure.

The line $a_{1}$ is in accordance with the principles stated above thought of as projected into the projection space and is drawn on its sagittal plane. This projection $p_{1}$ is given an arbitrary angle $\theta_{1}$ to the picture plane, and by this its length is determined. In the same way the projection of $a_{2}$ on the left side of the axis is drawn. However, according to the rule of invariance of length, the length of this projection is determined $\left(p_{2}=p_{1}\right)$. Thus, also the angle $\theta_{2}$ is determined and is dependent on the value chosen for $\theta_{1}$.

The angles $\beta_{1}$ and $\beta_{2}$ represent the visual angles for $a_{1}$ and $a_{2}$. Because $a_{1}$ and $a_{2}$ and also the distance $b$ are known in the experimental situation the angles $\beta_{1}$ and $\beta_{2}$ are given by:

$$
\begin{aligned}
& \tan \beta_{1}=a_{1} / b \\
& \tan \beta_{2}=a_{2} / b
\end{aligned}
$$

In the two upper triangles of the figure the following relations are valid in accordance with the law of sine:

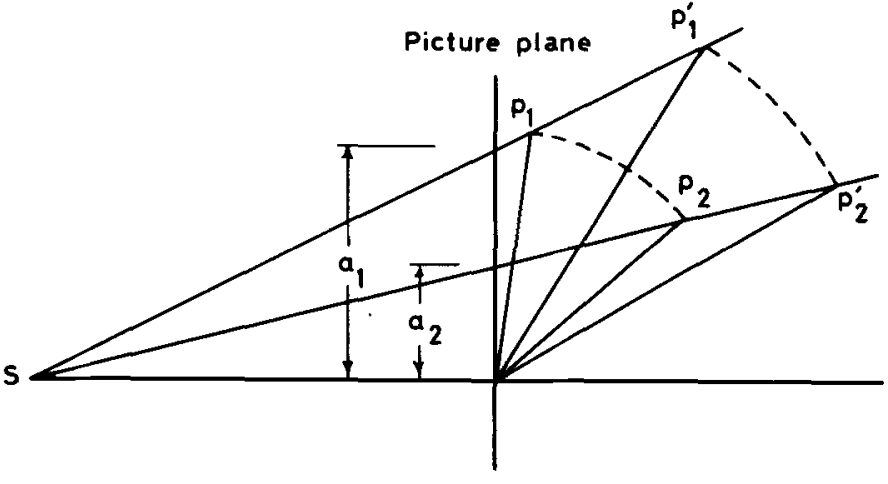

Fig. 3. Two examples ( $\left(P\right.$ and $\left.P^{\prime}\right)$ of possible projections when the line describes a rotary motion. $S$ is the station point of the projection system and a the proximal stimulus line in the picture plane. The number indices indicate two different lengths of the stimulus line and corresponding projections. 


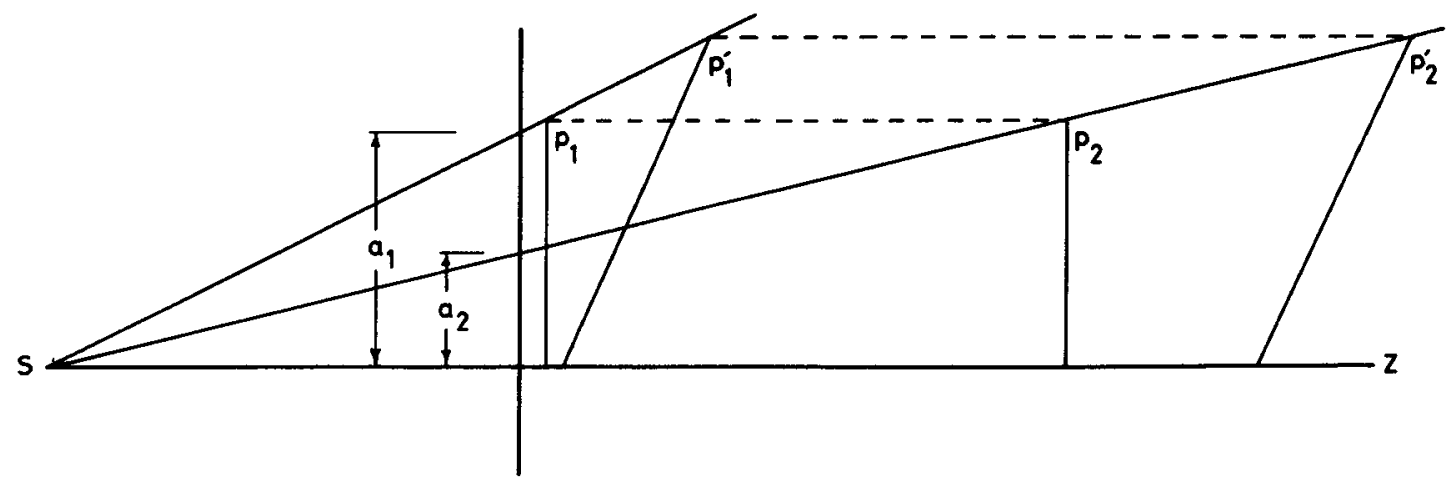

Fig: 4. Two examples ( $P$ and $\left.P^{\prime}\right)$ of possible projections when the line describes a translatory motion backwards in the projection space. Denotations as in Fig. 3.

$$
\frac{p_{1}}{\operatorname{sine}\left(90^{\circ}+\beta_{1}\right)}=\frac{a_{1}}{\operatorname{sine}\left(90^{\circ}-\theta_{1}-\beta_{1}\right)} \text {; and }
$$

$$
\frac{p_{2}}{\text { sine }\left(90^{\circ}+\beta_{2}\right)}=\frac{a_{2}}{\operatorname{sine}\left(90^{\circ}-\theta_{2}-\beta_{2}\right)}
$$

(3) and (4) are solved for $p_{1}$ and $p_{2}$, respectively, and as $p_{1}=p_{2}$ we get

$$
\begin{aligned}
& \frac{a_{1} \cos \beta_{1}}{\cos \left(\theta_{1}+\beta_{1}\right)}=\frac{a_{2} \cos \beta_{2}}{\cos \left(\theta_{2}+\beta_{2}\right)} ; \text { and } \\
& \cos \left(\theta_{2}+\beta_{2}\right)=\frac{a_{2} \cos \left(\theta_{1}+\beta_{1}\right) \cos \beta_{2}}{a_{1} \cos \beta_{1}}
\end{aligned}
$$

Because $\beta_{1}$ and $\beta_{2}$ are known, Equation (6) makes us able to compute the value of $\theta_{2}$ for any $\theta_{1}$ chosen and for any combination of $a_{1}$ and $a_{2}$.

In the experiment to be reported here all $\beta$-angles were $<1.6 \mathrm{deg}$. The accepted error in angle readings was of the order $\pm 2.5 \mathrm{deg}$. Under these conditions there exists no reason for maintaining, in our experiment and data treatment, the theoretically important demand for polar projection. Consequently, the computational formula could be simplified by dropping the $\beta$-terms;

$$
\cos \theta_{2}=\frac{a_{2} \cos \theta_{1}}{a_{1}}
$$

This equation is in fact Equation (6) adapted for parallel projection conditions.

The subliminal difference between the two-principles of projection under our conditions of visual angle also made it possible to apply parallel projection principles when constructing the stimulus patterns. This brought about an extremely impoverished stimulation, lacking the information about rigidity and direction inherent in a nonsymmetrical change in accordance with polar projection.

It may also be explicitly noted that the model does not specify anything about $\theta_{1}$, only about $\theta_{2}$ when $\theta_{1}$ is known. Thus, before any prediction from the model can be made, a value for $\theta_{1}$ must be determined in some way.

\section{Problem}

\section{EXPERIMENT}

The model proposed assumes specific percepts from continuous change of the length and of the direction of the stimulus line. Furthermore, it states a set of rules, yielding simple quantitative relations between stimulus change and perceived event. The experiment was designed to separate the perceptual effects of change of length and change of direction of the line.

The stimulus patterns chosen execute a repetitive oscillation between two turning points. Referring to the model given above the problem for this experiment may be stated in this way: If the empirical $\theta_{1}$-angle is known, to what extent can the $\theta_{2}$-angle in the perceived event, as indicated on a response bar, be predicted by the model? (The means of the angles are considered.)

\section{Stimulus Patterns}

A set of seven different stimulus patterns was prepared. These patterns are specified in Fig. 6. Patterns 1, 2, and 3 were designed for analyzing the effect of changing length of the stimulus line.

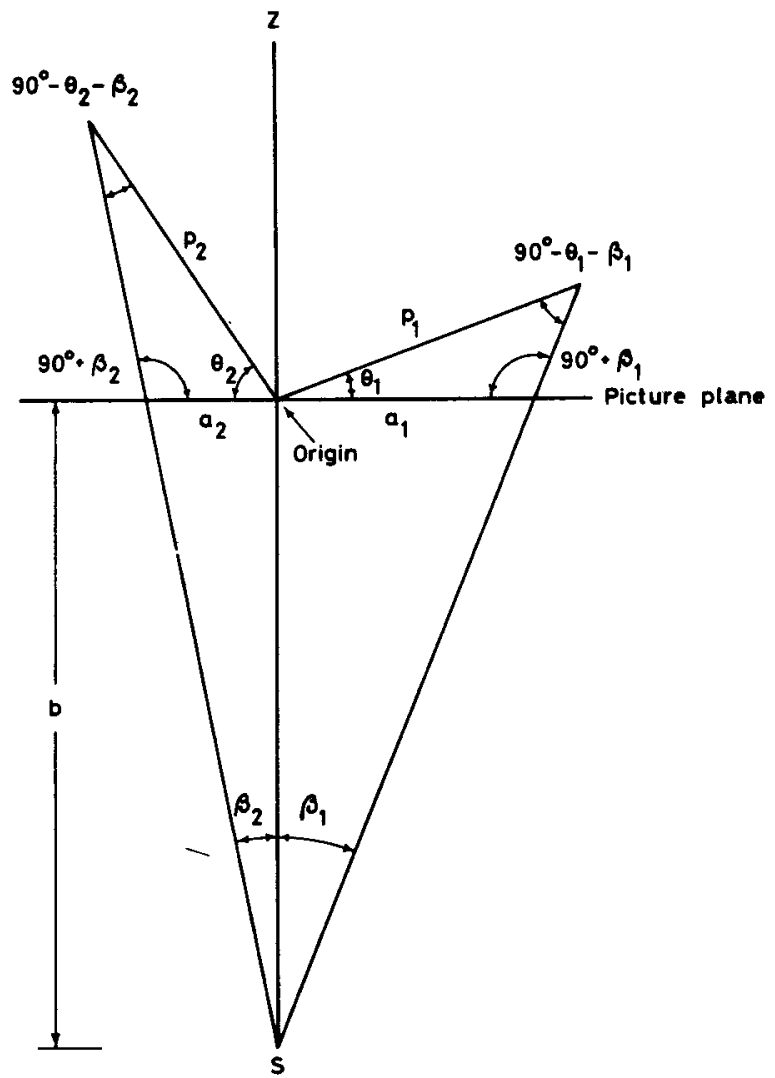

Fig. 5. A sagittal plane in the projection system with two turning positions of half of the stimulus line (for explanation see text). 

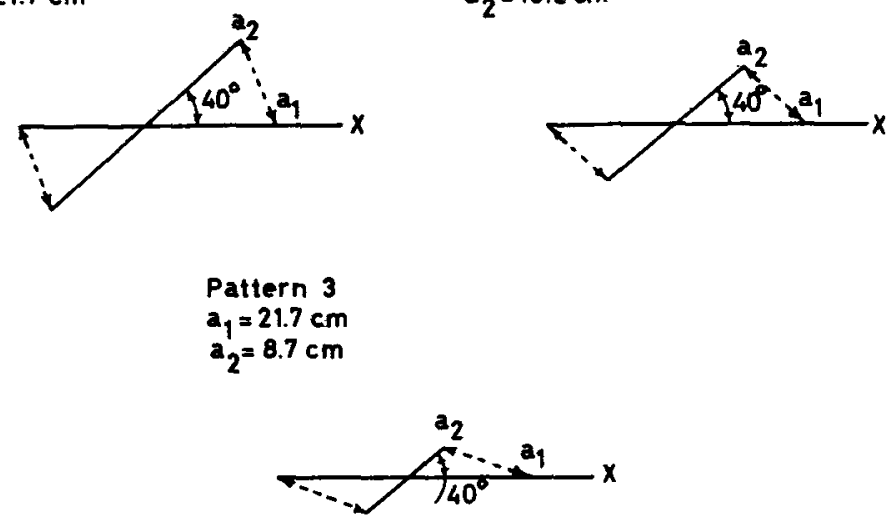
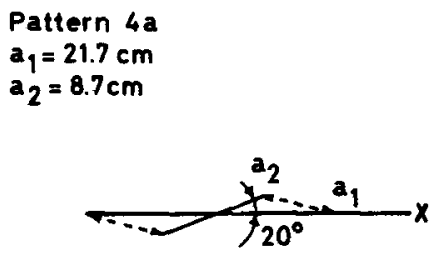

$$
\begin{aligned}
& \text { Pattern 4b } \\
& a_{1}=21.7 \mathrm{~cm} \\
& a_{2}=8.7 \mathrm{~cm}
\end{aligned}
$$

$$
\begin{aligned}
& a_{1}=21.7 \mathrm{~cm} \\
& a_{2}=8.7 \mathrm{~cm}
\end{aligned}
$$$$
5 a
$$

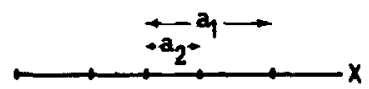

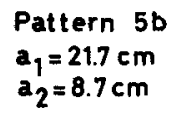

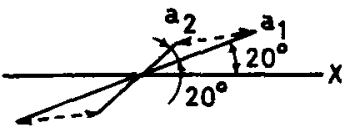

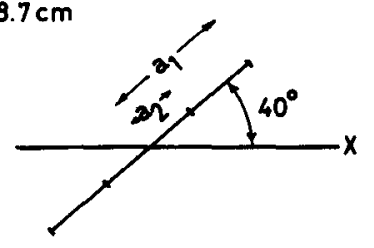

Fig. 6. Stimulus patterns. $a_{1}$ and $a_{2}$ are the upper half of the stimulus line in the two turning positions. The length of the line ctanged as shown by the dashed lines. At the S's eye the whole line subtended an angle of 1.2-3.1 deg.
The effect of changing rotation of the line was studied by the Patterns 3, 4(a and b), and 5(a and b). The two versions of Patterns 4 and 5 were included in order to check the effect of varying the orientation of the motion pattern on the picture plane. The rotation waveform was sinusoidal with a frequency of 0.2 cycles/sec. (A methodological experiment (Johansson \& Jansson, 1967) showed no effect of waveform used.)

\section{Stimulus Generation and Presentation}

The stimuli were produced by an animated film technique. Endless film loops containing a few numbers of full cycles of the periodic pattern were prepared. These film loops could be run in a film projector for an optional time. $16 \mathrm{~mm}$ film was used.

Two laboratory rooms were used. In one the stimulus patterns were projected with reduced brightness on a screen. This room was dark except for some stray light from the projector and from a small window in a door between the rooms. The $S$ sat in the other room in front of a table looking at the screen through the window of the door. This second room was enough lighted to make the first room appear, by brightness contrast, as a totally dark space. Under these conditions the stimulus line projected on the screen had no character of a film picture on a screen. Actually, it gave more the impression of a luminous object moving in a dark space. The distance from the $S$ to the window of the door was $3 \mathrm{~m}$ and from the $S$ to the screen $8 \mathrm{~m}$. The sound from the projector was masked.

Binocular vision was used because it was thought not to affect the percept because of the distance to the stimulation, but to increase the precision of the response (see below).

\section{Response Bar}

On the S's table a device was attached for indicating perceived directions or rotary motions of a rod. The principle for this device is shown in Fig. 7. The device was so adjusted that one of its axles was parallel to the sagittal axis from the S's eye to the center of the projection screen and the other axle had a frontal-parallel

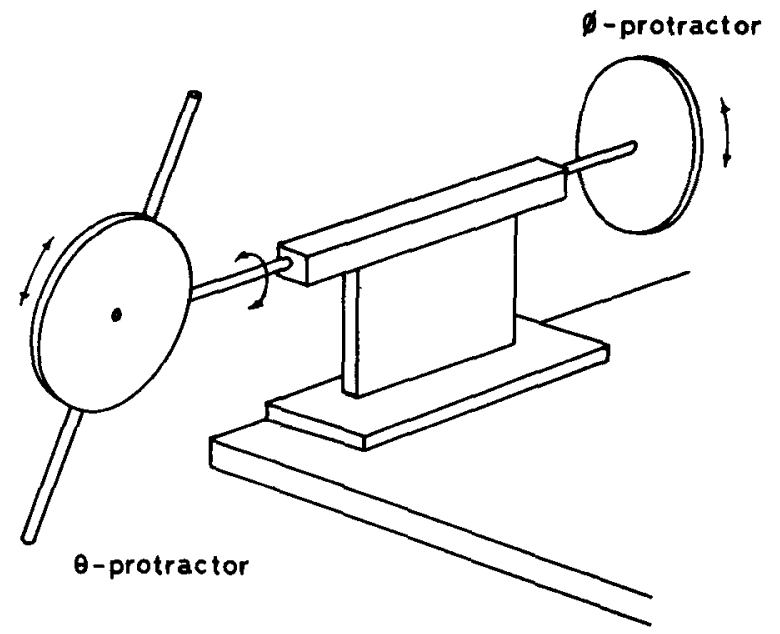

Fig. 7. The response bar. 
Table 1

Frequencies of the Five Categories of Verbal Descriptions

\begin{tabular}{crrrrrrrrrr}
\hline & & \multicolumn{10}{c}{ Stimulus pattern } \\
Category & 1 & 2 & 3 & $4 a$ & $4 b$ & $5 a$ & $5 b$ & Sum & $\%$ \\
1 & 43 & 2 & & & & 20 & 35 & 100 & 16 \\
2 & 40 & 82 & 85 & 76 & 80 & 56 & 60 & 479 & 77 \\
3 & 1 & & 3 & 8 & 13 & & 3 & 28 & 4 \\
4 & & & & & 1 & 15 & & 16 & 3 \\
5 & & & & & & 1 & 2 & 3 & 0 \\
Sum & 84 & 84 & 88 & 84 & 94 & 92 & 100 & 626 & 100 \\
\hline
\end{tabular}

orientation. Thus, on the protractor attached to the first mentioned axle the $\phi$-angle was read and the other protractor gave the value of the $\theta$-angle. The protractors were screened from the S's view.

\section{Procedure and Subjects}

The $S$ was seated at the table with the response bar close to his right hand. Before each trial the bar was brought to a vertical position. The stimulus pattern was shown, and the $S$ was asked to describe verbally what he saw. Provided the $\mathbf{S}$ had described some kind of rotary motion, he was asked to grip one end of the bar with his right hand and try to indicate the two turning positions, one by one. Each $S$ had five experimental sessions spread over five days with three blocks of the stimuli each session. Within the blocks the order of the stimuli was randomized. The Ss were allowed to report more than one percept for each stimulus, both verbally and with the response bar.

Ss were five undergraduate students of psychology.

\section{Results}

Verbal descriptions. The verbal descriptions were categorized in the following five categories:

(1) Two-dimensional changes in a frontal-parallel plane: the line either rotates or changes its length or executes both kinds of changes.

(2) Three-dimensional rotations with a fixed point as its center of rotation, the line having a constant length.

(3) The same kind of rotation as in Category (2), but the line changes also its length.

(4) Translatory motion of a line with constant length.

(5) The line, consisting of two straight parts, bends around its mid-point, and these parts rotate symmetrically on the same side of a frontal-parallel plane.

Category (1) here corresponds to Category (1) of the possible projections described above with the rotary component added when present. Categories (2) and (4) here correspond to Categories (2) and (3), respectively. Category (3) here is a subcategory to Category (5) there, and Category (5) here is not represented among the possible projections, because only straight projections are treated there.

The frequencies of these categories are given in Table 1 which shows that most of the descriptions belong to Category (2). Descriptions belonging to Category (1) appear mainly for the stimulus patterns which contain only one of the two types of changes. Ninety-eight per cent of these deseriptions are concentrated to Stimulus Patterns 1, 5a, and 5b. Most of the descriptions belonging to this category $(73 \%)$ are also concentrated to two of the Ss, one of which (S 5) also gave $91 \%$ of the descriptions belonging to Categories (3)-(5).

Thus, the majority (four Ss) had few or no descriptions belonging to Categories (3)-(5), and three of these Ss had also very few descriptions belonging to Category (1), and when these descriptions appeared they were concentrated to the stimulus patterns containing only one type of change.

Turning positions. The turning positions of the perceived movements could all be recorded by means of the response bar, except those belonging to Response Categories (4) and (5). Only the responses belonging to Categories (1)-(3) are therefore considered here. As is shown in Table 1 these categories include $97 \%$ of all the verbal descriptions.

The main interest is centered to the $\theta$-angles, defined as deviation from a frontal-parallel plane. When the upper part of the response bar was directed away from the $S$ the $\theta$-angle was given a positive sign, when it was directed towards the $S$ a negative sign.

In the treatment of the data no regard was taken to the sign of the $\theta_{2}$-angle, i.e., the angle corresponding to the shortest stimulus line. There was no significant difference between the data for the two main directions, and they are thus combined here. A positive $\theta_{2}$-angle was most common (67\% of the data).

In the treatment of the data for the $\theta_{1}$-angle regard was taken, however, to the sign of the angle, because these data were interpreted to be random values around zero.

The $\theta$-angles corresponding to the Response Categories (1)-(3) are given in Table 2 . The number of data differs between stimuli because the Ss were allowed to give more than one response. S 5 , who deviated most in her verbal descriptions, also deviated from the rest of the Ss when indicating the turning positions. Therefore her data are not included in the group data.

For Stimulus Patterns $5 \mathrm{a}$ and $5 \mathrm{~b}$ three of the four $\mathrm{Ss}$ in the majority group had a rather large difference between the $\theta$-angles, but $S 4$ yielded no difference for all responses except two. (The remaining two had a mean difference of $33 \mathrm{deg}$.) For Stimulus Patterns 5a and 5b the data are combined for Ss 1-3, and the data for $S 4$ are treated separately.

A comparison between Stimulus Patterns 1, 2, and 3 shows that amount of change of length is an important variable for amount of perceived movement in depth $\left(\theta_{2}-\theta_{1}\right)$. A comparison between stimulus Patterns 3, 4 ( $a$ and $b$ ), and 5 ( $a$ and $b$ ) shows that the amount of change of direction is of no or very little importance for the amount of perceived movement in depth. For one of the Ss (S 4) there must be some change of direction in order to get a perceived movement in depth. But also for this $S$ there was no difference between stimulus patterns with different amounts of change of direction above zero-change. A comparison between the a- and b-versions of stimulus Patterns 4 and 5 shows a very small

Table 2

The $\theta$-angles

\begin{tabular}{|c|c|c|c|c|c|c|c|c|c|c|c|c|c|c|c|}
\hline & & \multicolumn{14}{|c|}{ Stimulus pattern } \\
\hline & & \multicolumn{2}{|c|}{1} & \multicolumn{2}{|c|}{2} & \multicolumn{2}{|c|}{3} & \multicolumn{2}{|c|}{$4 a$} & \multicolumn{2}{|c|}{$4 b$} & \multicolumn{2}{|c|}{$5 a$} & \multicolumn{2}{|c|}{$5 b$} \\
\hline Subjects 1-4 & $\mathbf{M}$ & $\begin{array}{l}\theta_{1} \\
4\end{array}$ & $\begin{array}{l}\theta_{2} \\
19\end{array}$ & $\begin{array}{c}\theta_{1} \\
1\end{array}$ & $\begin{array}{l}\theta_{2} \\
36\end{array}$ & $\begin{array}{l}\theta_{1} \\
1\end{array}$ & $\begin{array}{l}\theta_{2} \\
50\end{array}$ & $\begin{array}{l}\theta_{1} \\
1\end{array}$ & $\begin{array}{l}\theta_{2} \\
51\end{array}$ & $\begin{array}{l}\theta_{1} \\
5\end{array}$ & $\begin{array}{c}\theta_{2} \\
51\end{array}$ & $\begin{array}{l}\theta_{\mathrm{t}} \\
1\end{array}$ & $\begin{array}{l}\theta_{2} \\
54\end{array}$ & $\begin{array}{l}\theta_{1} \\
1\end{array}$ & $\begin{array}{l}\theta_{2} \\
53\end{array}$ \\
\hline (patterns & $\sigma$ & 9 & 12 & 8 & 11 & 6 & 8 & 5 & 8 & 12 & 7 & 7 & 9 & 9 & 7 \\
\hline $\begin{array}{l}\text { Sa and b } \\
\text { only sub- } \\
\text { jects } 1-3 \text { ) }\end{array}$ & $\mathbf{n}$ & 60 & 69 & 60 & 79 & 60 & 87 & 60 & 92 & 60 & 92 & 49 & 87 & 47 & 78 \\
\hline Subject 4 & $\mathbf{M}$ & & & & & & & & & & & 2 & 2 & 0 & 0 \\
\hline (patterns & $\sigma$ & & & & & & & & & & & 2 & 2 & 3 & 3 \\
\hline $5 a$ and $b$ ) & $\mathrm{n}$ & & & & & & & & & & & 13 & 13 & 13 & 13 \\
\hline \multirow[t]{3}{*}{ Subject 5} & $\mathbf{M}$ & 3 & 6 & 2 & 14 & 1 & 23 & 1 & 18 & 5 & 19 & 0 & 0 & 4 & 7 \\
\hline & $\sigma$ & 3 & 5 & 2 & 6 & 2 & 6 & 2 & 6 & 5 & 8 & 0 & 0 & 5 & 5 \\
\hline & $\mathrm{n}$ & 15 & 15 & 15 & 15 & 15 & 15 & 15 & is & 15 & 15 & 15 & 15 & 15 & 16 \\
\hline
\end{tabular}


Table 3

The o-angles

\begin{tabular}{|c|c|c|c|c|c|c|c|c|c|c|c|c|c|c|c|}
\hline \multirow{5}{*}{$\begin{array}{r}\text { Subjects } 1-4 \\
\text { (patterns } \\
5 \text { a and b } \\
\text { only sub- } \\
\text { jects 1-3) }\end{array}$} & & \multicolumn{14}{|c|}{ Stimulus pattern } \\
\hline & & \multicolumn{2}{|c|}{1} & \multicolumn{2}{|c|}{2} & \multicolumn{2}{|c|}{3} & \multicolumn{2}{|c|}{$\mathbf{4 a}$} & \multicolumn{2}{|c|}{$4 \mathrm{~b}$} & \multicolumn{2}{|c|}{$5 \mathrm{a}$} & \multicolumn{2}{|c|}{$5 b$} \\
\hline & $\mathbf{M}$ & $\begin{array}{c}\phi_{1} \\
5\end{array}$ & $\begin{array}{l}\phi_{2} \\
48\end{array}$ & $\begin{array}{c}\phi_{1} \\
5\end{array}$ & $\begin{array}{c}\phi_{2} \\
50\end{array}$ & $\begin{array}{r}\phi_{1} \\
4\end{array}$ & $\begin{array}{l}\phi_{2} \\
49\end{array}$ & $\begin{array}{r}\phi_{1} \\
4\end{array}$ & $\begin{array}{l}\phi_{2} \\
32\end{array}$ & $\begin{array}{l}\phi_{1} \\
27\end{array}$ & $\begin{array}{l}\phi_{2} \\
50\end{array}$ & $\begin{array}{r}\phi_{1} \\
2\end{array}$ & $\begin{array}{r}\phi_{2} \\
5\end{array}$ & $\begin{array}{l}\phi_{1} \\
50\end{array}$ & $\begin{array}{l}\phi_{2} \\
49\end{array}$ \\
\hline & $\sigma$ & 2 & 9 & 7 & 8 & 5 & 11 & 5 & 12 & 4 & 13 & 3 & 8 & 6 & 14 \\
\hline & $\mathbf{n}$ & 60 & 69 & 60 & 79 & 60 & 87 & 60 & 92 & 60 & 92 & 49 & 87 & 47 & 78 \\
\hline Subject 4 & $\mathbf{M}$ & & & & & & & & & & & 0 & 0 & 45 & 45 \\
\hline (patterns & 0 & & & & & & & & & & & 0 & 0 & 2 & 2 \\
\hline Sa and b) & $\mathrm{n}$ & & & & & & & & & & & 13 & 13 & 15 & 15 \\
\hline Subject 5 & $\mathbf{M}$ & 2 & 48 & 4 & 47 & 3 & 45 & 2 & 28 & 29 & 49 & 1 & 1 & 50 & 49 \\
\hline & $\sigma$ & 2 & 4 & 2 & 5 & 2 & 6 & 3 & 4 & 3 & 4 & 2 & 2 & 3 & 3 \\
\hline & $\mathbf{n}$ & 15 & 15 & 15 & 15 & 15 & 15 & 15 & 15 & 15 & 15 & 15 & 15 & 15 & 16 \\
\hline
\end{tabular}

effect, if any, of the general orientation of the pattern.

The $\phi$-angles are given in Table 3 . It may be observed that there is a slight but systematic overestimation of the difference between the $\phi$-angles $\left(\phi_{2}-\phi_{1}\right)$.

The differences between the predicted and the empirical $\theta_{2}$-angles are given in Table 4.

\section{DISCUSSION}

The experiment reported here shows that there is a dominant tendency to perceive stimuli of the kind used as moving into the third dimension. The same tendency was shown in two other experiments reported elsewhere (Johansson \& Jansson, 1967). In these two experiments together with the one described here $38 \mathrm{Ss}$ took part. About $80 \%$ of all the responses in all the experiments were three-dimensional. Most of the remaining $20 \%$ were concentrated to a few Ss. The basic principle in the model is thus generally verified, at least approximately.

The model predicts that amount of change of length determines the perceived movement in depth and that amount of change of direction determines the perceived change of direction, but not the perceived movement in depth. The general orientation of the motion pattern is unimportant according to the model. For the majority of the data in this experiment these general predictions are verified.

However, there are two kinds of deviations from the predicted values. The movement in depth for Stimulus Pattern 1 was not predicted because this pattern has no change in length. At the present stage of the investigation we have to accept it, but it ought to be studied further. It may be that it is a rather trivial effect from the experimental arrangement.

The other deviation from the model is the amount of perceived movement in depth which deviates from the predicted values as shown in Table 4. Several hypotheses are available to explain this result. The concentration of the two-dimensional responses in a few . Ss may indicate that these Ss did not have the minimum of some perceptual skill required by this impoverished stimulation. It can be hypothesized that also these Ss would give three-dimensional responses, if more information was added to the stimulation

Table 4

Differences Between Predicted and Empirical Mean

Values of $\theta_{2}$, the Predictions Made from the Means of $\theta_{1}$ with Formula (7)

\begin{tabular}{|c|c|c|c|c|c|c|c|}
\hline \multirow[b]{3}{*}{$\begin{array}{l}\text { Subjects } 1-4 \\
\text { (patterns } \\
5 a \text { and b only } \\
\text { subjects } 1-3 \text { ) }\end{array}$} & \multicolumn{7}{|c|}{ Stimulus pattern } \\
\hline & 1 & 2 & 3 & $4 a$ & $4 b$ & $5 a$ & $5 b$ \\
\hline & -15 & 10 & 16 & 15 & 15 & 12 & 13 \\
\hline $\begin{array}{l}\text { Subject } 4 \\
\text { (pattern } 5 a \\
\text { and b) }\end{array}$ & & & & & & 64 & 66 \\
\hline Subject 5 & -3 & 32 & 43 & 43 & 47 & 66 & 63 \\
\hline
\end{tabular}

(cf., Green, 1961). Another possibility would be to question some assumptions in the model, mainly the assumption about the perceived rigidity of the "rod." These explanatory attempts are ex post facto, however, and ought to be studied further.

In another respect our experiments have provided material for an important further specification of the model. In these experiments the visual system has applied the rule that one of the two turning positions was seen as parallel to our hypothetical frontal plane. In this rather unexpected way the human visual system gets an intraand also inter-individual specificity from unspecific stimulation. (The slight but systematic deviation from exact frontal-parallelity found may be due to the actual experimental arrangements, and is therefore of limited interest from a theoretical point of view.) It seems very plausible that this preference for frontal-parallel orientation, when every cue for specific orientation is lacking, is related to the "tendency to equidistance" investigated by Gogel (1965). Thus, we will add to our model as a third restricting principle for decoding our stimulus patterns the following statement, valid for stimulus patterns lacking any other specific information about direction: The line will always be seen in a frontal-parallel direction in the moment when it has its maximal extension.

\section{REFERENCES}

BERKELEY, G. An essay towards a new theory of vision, 1709. Also in A. A. Luce and T. E. Jessop (Eds.), The works of George Berkeley, Bishop of Cloyne. New York: Nelson, 1948.

BRAUNSTEIN, M. L. Depth perception in rotating dot patterns: effects of numerosity and perspective. J. exp. Psychol, 1962, 64, 415-420.

GIBSON, J. J., \& GIBSON, E. J. Continuous perspective transformations and the perception of rigid motion. J. exp. Psychol, 1957, 54, 129-138.

GOGEL, W. C. Equidistance tendency and its consequences. Prychol Bull, $1965,64,153-163$.

GREEN, B. G. Figure coherence in the kinetic depth effect. J. exp. Psychol., $1961,62,272-282$.

JOHANSSON, G. Perception of motion and changing form. Scand. $J$. Pyychol, 1964, 5, 181-208.

JOHANSSON, G., \& JANSSON, G. A model for space perception and object perception from changes in a straight line. 41 st report, Department of Psychology, University of Uppsala, 1967.

WALLACH, H., \& O'CONNELL, D. N. The kinetic depth effect. J. exp. Psychol, 1953, 45, 205-217.

WHITE, B. W., \& MUESER, G. E. Accuracy in reconstructing the arrangement of elements generating kinetic depth displays. $J$. exp. Psychol, 1960, 60, 1-11.

\section{NOTES}

1. The authors are indebted to Mrs. Kristina Israelsson for her experimental and computational work, and to Dr. William Epstein for his highly valuable comments on the manuscript. The experimental investigation was made pos sible by grants from the Swedish Council for Social Science Research, and the theoretical work was finished during the senior writer's fellowship year at the Center for Advanced Study in the Behavioral Sciences, Stanford.

2. Address: Department of Psychology, S:t Larsgatan 2, S-75220 Uppsala, Sweden.

(Accepted for publication May 7, 1968.) 